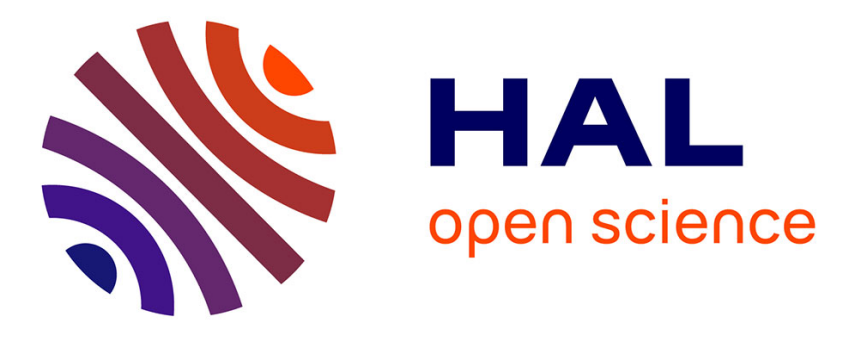

\title{
The symmetric BEM: bringing in more variables for better accuracy
}

Maureen Clerc, Alexandre Gramfort, Emmanuel Olivi, Théodore Papadopoulo

\section{To cite this version:}

Maureen Clerc, Alexandre Gramfort, Emmanuel Olivi, Théodore Papadopoulo. The symmetric BEM: bringing in more variables for better accuracy. Biomag 2010, S. Supek, May 2010, Dubrovnik, Croatia. pp.109-112, 10.1007/978-3-642-12197-5_21 . inria-00497081

\section{HAL Id: inria-00497081 \\ https://hal.inria.fr/inria-00497081}

Submitted on 2 Jul 2010

HAL is a multi-disciplinary open access archive for the deposit and dissemination of scientific research documents, whether they are published or not. The documents may come from teaching and research institutions in France or abroad, or from public or private research centers.
L'archive ouverte pluridisciplinaire HAL, est destinée au dépôt et à la diffusion de documents scientifiques de niveau recherche, publiés ou non, émanant des établissements d'enseignement et de recherche français ou étrangers, des laboratoires publics ou privés. 


\title{
The symmetric BEM: bringing in more variables for better accuracy
}

\author{
Maureen Clerc $^{1}$, Alexandre Gramfort ${ }^{1,2}$, Emmanuel Olivi $^{1}$ and Theo Papadopoulo ${ }^{1}$ \\ ${ }^{1}$ INRIA Odyssée Project-Team, Sophia Antipolis, France \\ ${ }^{2}$ INRIA Parietal Project Team, Saclay, France
}

\begin{abstract}
Electrophysiological modeling of Magneto- and Electro-encephalography (MEG and EEG) rely on accurate forward solvers that relate source activities to sensor measurements. In comparing a Boundary Element (BEM) and a Finite Element Method (FEM) for forward electroencephalography, in our early numerical experiments, we found the FEM to have a better accuracy than the BEM. This triggered a quest to improve the accuracy of Boundary Element Methods and led us to study the extended Green representation theorem.

A fundamental result in potential theory shows that, up to an additive constant, a harmonic function is determined within a domain from its value on the boundary (Dirichlet condition), or the value of its normal derivative (Neumann condition). The Green Representation Theorem has been used in forward EEG and MEG modeling, in deriving the Geselowitz BEM formulation, and the Isolated Problem Approach. The extended Green Representation Theorem provides a representation for the directional derivatives of a piecewise-harmonic function.

By introducing the normal current as an additional variable in the forward problem, we derive a new Boundary Element Method, which leads to a symmetric matrix structure: we hence call it the Symmetric BEM. Accuracy comparisons demonstrate the superiority of the Symmetric BEM to the FEM and to the classical BEM.
\end{abstract}

Keywords - Boundary Element Method, Electroencephalography, Magnetoencephalography, Forward Problem.

\section{INTRODUCTION}

Electrophysiological modeling of MEG and EEG rely on forward solvers that relate the source activity to the sensor measurements. In comparing a Boundary Element Method (BEM) and a Finite Element Method (FEM) for forward electroencephalography, in our early numerical experiments, we found a superior accuracy to the FEM [1]. This triggered a quest to improve the accuracy of Boundary Element Methods and led us to study the extended Green representation theorem [2]. We proposed a common formalism for the integral formulations of the forward EEG problem, and we derived three different Boundary Element Methods within the same framework [3]. In this paper we recall the mathematical background of Boundary Element Methods, and we present both the double-layer BEM, which is the most widespread method, and the Symmetric BEM, which is a new formulation. Numerical comparisons on a three-layer spherical model show the superiority of the Symmetric BEM in terms of accuracy, compared to the double-layer BEM.

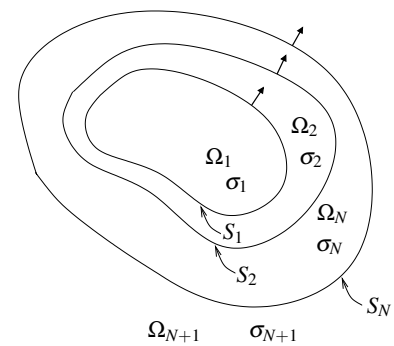

Fig. 1: Boundary Elements are well-suited for piecewise constant isotropic conductivity models. The head, denoted $\Omega$, is here modeled as a set of nested domains $\Omega_{i}, i=1 \ldots N$, each with a constant conductivity $\sigma_{i}$. The outer domain $\Omega_{N+1}$, representing the air, is non-conductive $\left(\sigma_{N+1}=0\right)$.

\section{GREEN REPRESENTATION}

A fundamental result in potential theory shows that, up to an additive constant, a harmonic function (i.e., such that $\Delta u=0$ ) is uniquely determined within a domain $\Omega$ from its value on the boundary $\partial \Omega$ (Dirichlet condition), or the value of its normal derivative (Neumann condition). The Green Representation Theorem gives an explicit representation of a piecewise-harmonic function as a combination of boundary integrals of its jumps and the jumps of its normal derivative across interfaces. Before stating this theorem, some notation must be defined:

- The restriction of a function $f$ to a surface $S_{j}$ is indicated by $f_{S_{j}}$.

- The functions $f_{S_{j}}^{-}$and $f_{S_{j}}^{+}$represent the interior and exterior limits of $f$ on $S_{j}$ :

$$
\text { for } \mathbf{r} \in S_{j}, \quad f_{S_{j}}^{ \pm}(\mathbf{r})=\lim _{\alpha \rightarrow 0^{ \pm}} f(\mathbf{r}+\alpha \mathbf{n}) .
$$

- The jump of a function $f$ across $S_{j}$ is denoted by:

$$
[f]_{S_{j}}=f_{S_{j}}^{-}-f_{S_{j}}^{+},
$$


- $\partial_{\mathbf{n}} V=\mathbf{n} \cdot \nabla V$ denotes the partial derivative of $V$ in the direction of a unit vector $\mathbf{n}$,

- The function $G(\mathbf{r})=\frac{1}{4 \pi\|\mathbf{r}\|}$ is the fundamental solution of the Laplacian in $\mathbb{R}^{3}$, such that $-\Delta G=\delta_{0}$.

Consider an open region $\Omega$ and a function $u$ such that $\Delta u=$ 0 in $\Omega$ and in $\mathbb{R}^{3} \backslash \Omega$ (but not necessarily continuous across $\partial \Omega$ ). The Green Representation Theorem states that, for a point $\mathbf{r}$ belonging to $\partial \Omega$,

$$
\begin{aligned}
\frac{u^{-}(\mathbf{r})+u^{+}(\mathbf{r})}{2}=-\int_{\partial \Omega}[u] & \partial_{\mathbf{n}^{\prime}} G\left(\mathbf{r}-\mathbf{r}^{\prime}\right) d s\left(\mathbf{r}^{\prime}\right) \\
& +\int_{\partial \Omega}\left[\partial_{\mathbf{n}^{\prime}} u\right] G\left(\mathbf{r}-\mathbf{r}^{\prime}\right) d s\left(\mathbf{r}^{\prime}\right) .
\end{aligned}
$$

This representation also holds for the head model in Figure 1, when $\Omega$ is the union of disjoint open sets: $\Omega=\Omega_{1} \cup \Omega_{2} \cup$ $\ldots \Omega_{N}$, with $\partial \Omega=S_{1} \cup S_{2} \cup \ldots S_{N}$. If $u$ is harmonic in each $\Omega_{i}$, for $\mathbf{r} \in S_{i}$,

$$
\begin{aligned}
\frac{u^{-}(\mathbf{r})+u^{+}(\mathbf{r})}{2}=-\sum_{j=1}^{N} \int_{S_{j}}[ & u]_{S_{j}} \partial_{\mathbf{n}^{\prime}} G\left(\mathbf{r}-\mathbf{r}^{\prime}\right) d s\left(\mathbf{r}^{\prime}\right) \\
& +\int_{S_{j}}\left[\partial_{\mathbf{n}^{\prime}} u\right]_{S_{j}} G\left(\mathbf{r}-\mathbf{r}^{\prime}\right) d s\left(\mathbf{r}^{\prime}\right)
\end{aligned}
$$

The notation is simplified by introducing two integral operators, which map a scalar function $f$ on $\partial \Omega$ to another scalar function on $\partial \Omega$ : the "double-layer" operator $(\mathcal{D} f)(\mathbf{r})=\int_{\partial \Omega} \partial_{\mathbf{n}^{\prime}} f\left(\mathbf{r}^{\prime}\right) G\left(\mathbf{r}-\mathbf{r}^{\prime}\right) d s\left(\mathbf{r}^{\prime}\right)$, and the "single-layer" operators $(\mathcal{S} f)(\mathbf{r})=\int_{\partial \Omega} f\left(\mathbf{r}^{\prime}\right) G\left(\mathbf{r}-\mathbf{r}^{\prime}\right) d s\left(\mathbf{r}^{\prime}\right)$. The restriction $\mathcal{D}_{i j}$ of an operator $\mathcal{D}$ maps a function of $S_{j}$ to a function of $S_{i}$.

An extension of the Green Representation Theorem represents the directional derivative of a harmonic function as a combination of boundary integrals of higher order. This requires two more integral operators: the adjoint $\mathcal{D}^{*}$ of the double-layer operator, such that

$$
\int_{S} \mathcal{D}^{*} f(\mathbf{r}) g(\mathbf{r}) d s(\mathbf{r})=\int f(\mathbf{r}) \mathcal{D} g(\mathbf{r}) d s(\mathbf{r}),
$$

and a hyper-singular operator $\mathcal{N}$ defined by $(\mathcal{N} f)(\mathbf{r})=$ $\int_{\partial \Omega} f\left(\mathbf{r}^{\prime}\right) \partial_{\mathbf{n}, \mathbf{n}^{\prime}}^{2} G\left(\mathbf{r}-\mathbf{r}^{\prime}\right) d s\left(\mathbf{r}^{\prime}\right)$. For a point $\mathbf{r}$ belonging to $\partial \Omega$,

$$
-\frac{\partial_{\mathbf{n}} u^{-}(\mathbf{r})+\partial_{\mathbf{n}} u^{+}(\mathbf{r})}{2}=+\mathcal{N}[u]-\mathcal{D}^{*}\left[\partial_{\mathbf{n}} u\right]
$$

and for a nested head model, if $\mathbf{r}$ is a point of $S_{i}$,

$$
-\frac{\partial_{\mathbf{n}} u^{-}(\mathbf{r})+\partial_{\mathbf{n}} u^{+}(\mathbf{r})}{2}=\sum_{j=1}^{N} \mathcal{N}_{i j}[u]_{S_{j}}-\sum_{j=1}^{N} \mathcal{D}_{j i}^{*}\left[\partial_{\mathbf{n}} u\right]_{S_{j}}
$$

As we shall see in the next section, the Geselowitz formula exploits only the first boundary integral representation equation (2), while it is possible to exploit both (2) and (3). Thus, three Boundary Element Methods can be derived within a unified setting: a BEM involving only single-layer potentials, a BEM involving only double-layer potentials, and a BEM combining single- and double-layer potentials [3]. We concentrate hereforth on the latter two BEMs.

\section{FORWARD EEG AND MEG}

To apply the representation theorem to the forward problem of EEG, a harmonic function must be produced, which relates the potential and the sources.

\section{A. The double-layer BEM}

Decomposing the source term as $f=\sum_{i} f_{i}$ where the support of each $f_{i}$ lies inside $\Omega_{i}$, consider $v_{\Omega_{i}}$ such that $\Delta v_{\Omega_{i}}=f_{i}$ holds in all $\mathbb{R}^{3}$. The function $v_{d}=\sum_{i=1}^{N} v_{\Omega_{i}}$ satisfies $\Delta v_{d}=f$ and is continuous across each surface $S_{i}$, as well as its normal derivative $\partial_{\mathbf{n}} v_{d}$. The function $u=\sigma V-v_{d}$ is a harmonic function in $\Omega$, to which (2) can be applied. Since $[u]_{S_{i}}=\left(\sigma_{i}-\sigma_{i+1}\right) V_{j}$ and $\left[\partial_{\mathbf{n}} u\right]=0$, we obtain, on each surface $S_{i}$,

$$
\frac{\sigma_{i}+\sigma_{i+1}}{2} V_{j}+\sum_{j=1}^{N}\left(\sigma_{j}-\sigma_{j+1}\right) \mathcal{D}_{i j} V_{j}=v_{d} .
$$

The above formula was established by Geselowitz [4], and was the only one used to model electroencephalography or electrocardiography, until recently, when [3] showed the diversity of BEMs that can be derived. This classical BEM is called a double-layer BEM because it only involves the double-layer operator $\mathcal{D}$.

As such, the double-layer BEM shows poor accuracy when there are large variations in conductivity between neighboring regions (such as the skull and the scalp). For this, a modified formulation, called the Isolated Problem Approach (or alternatively, Isolated Skull Approach), has been proposed [5]. This formulation is the most widespread in the forward BEM, and will be used in Numerical Results Section IV.

\section{B. The Symmetric BEM}

The originality of the Symmetric Boundary Element Method is to consider a different piecewise harmonic function for each domain $\Omega_{i}$ : a function $u_{\Omega_{i}}$ equal to $V-\frac{v_{\Omega_{i}}}{\sigma_{i}}$ 
within $\Omega_{i}$ and to $-\frac{v_{\Omega_{i}}}{\sigma_{i}}$ outside of $\Omega_{i}$. This $u_{\Omega_{i}}$ is indeed harmonic in $\mathbb{R}^{3} \backslash \partial \Omega_{i}$, and the representation equations (2) and (3) can be applied, leading to a system of integral equations involving two types of unknowns: the potential $V_{S_{i}}$ and the normal current $p_{S_{i}}=\left(\sigma \partial_{\mathbf{n}} V\right)_{i}$ on each interface.

Evaluating the internal limit of $u_{\Omega_{i}}$ on $S_{i}$ and the external limit of $\Omega_{i+1}$ on $S_{i}$ via the extended Representation Theorem, and subtracting the two representations gives, for $i=1, \ldots, N$ :

$$
\begin{aligned}
\sigma_{i+1}^{-1}\left(v_{\Omega_{i+1}}\right)_{S_{i}} & -\sigma_{i}^{-1}\left(v_{\Omega_{i}}\right)_{S_{i}}= \\
\mathcal{D}_{i, i-1} V_{S_{i-1}} & -2 \mathcal{D}_{i i} V_{S_{i}}+\mathcal{D}_{i, i+1} V_{S_{i+1}}-\sigma_{i}^{-1} \mathcal{S}_{i, i-1} p_{S_{i-1}} \\
& +\left(\sigma_{i}^{-1}+\sigma_{i+1}^{-1}\right) \mathcal{S}_{i i} p_{S_{i}}-\sigma_{i+1}^{-1} \mathcal{S}_{i, i+1} p_{S_{i+1}} .
\end{aligned}
$$

Using the same approach, the quantities $\left(\sigma_{i} \partial_{\mathbf{n}} u_{\Omega_{i}}\right)_{S_{i}}^{-}=$ $\left(p-\partial_{\mathbf{n}} v_{\Omega_{i}}\right)_{S_{i}}^{-}$and $\left(\sigma_{i+1} \partial_{\mathbf{n}} u_{\Omega_{i+1}}\right)_{S_{i}}^{+}=\left(p-\partial_{\mathbf{n}} v_{\Omega_{i+1}}\right)_{S_{i}}^{+}$can be evaluated using the extended Representation Theorem. Subtracting the two representations yields

$$
\begin{aligned}
&\left(\partial_{\mathbf{n}} v_{\Omega_{i+1}}\right)_{S_{i}}-\left(\partial_{\mathbf{n}} v_{\Omega_{i}}\right) S_{i}= \\
& \sigma_{i} \mathcal{N}_{i, i-1} V_{S_{i-1}}-\left(\sigma_{i}+\sigma_{i+1}\right) \mathcal{N}_{i i} V_{S_{i}}+\sigma_{i+1} \mathcal{N}_{i, i+1} V_{S_{i+1}}- \\
& \mathcal{D}_{i, i-1}^{*} p_{S_{i-1}}+2 \mathcal{D}_{i i}^{*} p_{S_{i}}-\mathcal{D}_{i, i+1}^{*} p_{S_{i+1}}
\end{aligned}
$$

\section{Discretization}

The surfaces are represented by triangular meshes. To fix ideas, consider a three-layer model for the head. Conductivities of each domain are respectively denoted $\sigma_{1}, \sigma_{2}$ and $\sigma_{3}$. The surfaces enclosing these homogeneous conductivity regions are denoted $S_{1}$ (inner skull boundary), $S_{2}$ (skull-scalp interface) and $S_{3}$ (scalp-air interface).

The Symmetric BEM is discretized with with mixed P1P0 boundary elements. Surfacic P1 functions are continuous over their support and piecewise linear: they are linear on each triangle. Surfacic P0 functions are discontinuous over their support and piecewise constant: they are constant on each triangle. We denote $\psi_{i}^{(k)}$ the P0 boundary element associated to triangle $i$ on surface $S_{k}$ (equal to 1 on triangle $i$ and to 0 elsewhere), and $\phi_{j}^{(l)}$ the P1 boundary element associated to node $j$ on surface $S_{l}$ (equal to 1 at node $j$, linear on all triangles that touch node $j$ and equal to zero elsewhere). The potential $V$ on surface $S_{k}$ is approximated by $V_{S_{k}}(\mathbf{r})=\sum_{i} x_{i}^{(k)} \phi_{i}^{(k)}(\mathbf{r})$, while $p=\sigma \partial_{\mathbf{n}} V$ on surface $S_{k}$ is approximated by $p_{S_{k}}(\mathbf{r})=\sum_{i} y_{i}^{(k)} \psi_{i}^{(k)}(\mathbf{r})$. The reason for discretizing $\sigma \partial_{\mathbf{n}} V$ with $\mathrm{P} 0$ elements while $V$ is discretized with $\mathrm{P} 1$ elements is that the gradient of a piecewise-linear function is piecewise constant.

As an illustration, consider the source term to reside in the brain compartment $\Omega_{1}$. After discretizing the equations (6) and (5), the variables $\left(\mathbf{x}_{k}\right)_{i}=x_{i}^{(k)}$ and $\left(\mathbf{y}_{k}\right)_{i}=y_{i}^{(k)}$ satisfy the linear system:

$$
\begin{aligned}
& {\left[\begin{array}{ccccc}
\left(\sigma_{1}+\sigma_{2}\right) \mathrm{N}_{11} & -2 \mathrm{D}_{11}^{*} & -\sigma_{2} \mathrm{~N}_{12} & \mathrm{D}_{12}^{*} & 0 \\
-2 \mathrm{D}_{11} & \left(\sigma_{1}^{-1}+\sigma_{2}^{-1}\right) \mathrm{S}_{11} & \mathrm{D}_{12} & -\sigma_{2}^{-1} \mathrm{~S}_{12} & 0 \\
-\sigma_{2} \mathrm{~N}_{21} & \mathrm{D}_{21}^{*} & \left(\sigma_{2}+\sigma_{3}\right) \mathrm{N}_{22} & -2 \mathrm{D}_{22}^{*} & -\sigma_{3} \mathrm{~N}_{23} \\
\mathrm{D}_{21} & -\sigma_{2}^{-1} \mathrm{~S}_{21} & -2 \mathrm{D}_{22} & \left(\sigma_{2}^{-1}+\sigma_{3}^{-1}\right) \mathrm{S}_{22} & \mathrm{D}_{23} \\
0 & 0 & -\sigma_{3} \mathrm{~N}_{32} & \mathrm{D}_{32}^{*} & \sigma_{3} \mathrm{~N}_{33}
\end{array}\right]\left[\begin{array}{l}
\mathbf{x}_{1} \\
\mathbf{y}_{1} \\
\mathbf{x}_{2} \\
\mathbf{y}_{2} \\
\mathbf{x}_{3}
\end{array}\right]} \\
& =\left[\begin{array}{c}
\mathbf{b}_{1} \\
\mathbf{c}_{1} \\
0 \\
0 \\
0
\end{array}\right]
\end{aligned}
$$

where $\mathbf{b}_{1}$ and $\mathbf{c}_{1}$ are the coefficients of the P0 (resp. P1) boundary element decomposition of the source term $\partial_{\mathbf{n}} v_{\Omega_{1}}$ (resp. $-\sigma_{1}^{-1} v_{\Omega_{1}}$ ).

Blocks $\mathrm{N}_{i j}$ and $\mathrm{D}_{i j}$ map a potential $V_{j}$ on $S_{j}$ to a function defined on $S_{i}$. Block $S_{i j}$ maps a normal current $p_{j}$ on $S_{j}$ to a function defined on $S_{i}$. The resulting matrix is block-diagonal, and symmetric, whence the name "Symmetric BEM".

The Symmetric BEM introduces an additional unknown into the problem: the normal current, and uses an additional set of representation equations linking the normal current and the potential. The Symmetric BEM departs from the doublelayer BEM in several ways:

- the normal current to each surface is explicitely modeled;

- only the surfaces which bound a common compartment have an interaction (whence the blocks of zeros in the matrix);

- only the surfaces which bound a compartment containing sources have a source term (whence the zeros in the righthand side vector);

- the design matrix is symmetric;

- the design matrix is larger than that of the double-layer BEM for a given head model, because there are more unknowns.

The magnetic field is computed from the electric field and the primary source distribution using the Biot and Savart equation, as proposed by Ferguson, Zhang and Stroink [6]. Two extensions of the Symmetric BEM have been proposed: the Symmetric BEM has been extended to non-nested geometries [8], and Fast Multipoles have been introduced in order to accelerate matrix-vector products for the inversion of the design matrix [7].

\section{NUMERICAL RESULTS}

The accuracy of a numerical method is measured by comparison with analytical solution, by means of the Relative 
relative difference (topographic) error

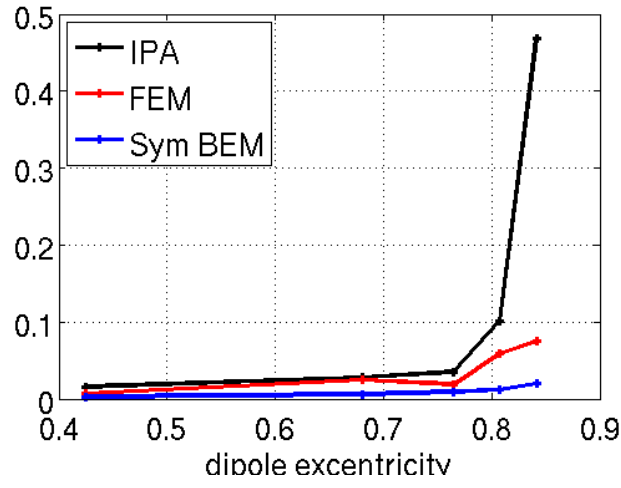

Fig. 2: Relative Difference Measure (RDM)

Difference Measure (RDM), defined by

$R D M\left(g_{\text {numeric }}, g_{\text {analytic }}\right)=\left\|\frac{g_{\text {numeric }}}{\left\|g_{\text {numeric }}\right\|}-\frac{g_{\text {analytic }}}{\left\|g_{\text {analytic }}\right\|}\right\| \in[0,2]$.

The analytical solution was computed, for a classical, threeshell spherical head model [9]. The spheres had radii 0.87, 0.92 and 1 and were each triangulated with 642 points. The conductivities of the three domains was set to $1,1 / 80$ and 1 . The volumes within the spheres were meshed with tetrahedra, which were designed to share the same vertices on the spheres as the ones used for the triangulations.

Figure 2 compares the accuracy of the Symmetric BEM (in blue) to a P1 tetrahedric Finite Element method (in red), and to a double-layer, IPA-corrected double-layer BEM (in black). The accuracy is here displayed for 5 dipoles on the zaxis with eccentricities $0.425,0.68,0.765,0.8075$ and 0.8415 and moment $(1,0,1)$. Though the accuracy of the Finite Element method is higher than that of the IPA-corrected doublelayer BEM, as initially shown in [1], the Symmetric BEM outperforms both methods.

\section{DISSEMINATION}

The Symmetric BEM is made available through the opensource code OpenMEEG (http://openmeeg.gforge.inria.fr). This C++ code can used either standalone, through a command-line interface or via Python, and its integration into standard EEG and MEG packages is under way (e.g., Fieldtrip).

\section{CONCLUSION}

By studying the extended Green Representation Theorem, several Boundary Element Methods for the forward EEG and MEG problems have been proposed. The most accurate among these is the Symmetric BEM. This increased accuracy for a given head model comes with the price of having more boundary integral operators to compute, and a larger design matrix to assemble. Interested readers may refer to [3] for more details on the method, and to http://openmeeg.gforge.inria.fr where an opensource implementation is available for download.

\section{REFERENCES}

1. Clerc M., Dervieux A., Faugeras O., Keriven R., Kybic J., Papadopoulo T.. Comparison of BEM and FEM methods for the E/MEG problem in Proceedings of BIOMAG 20022002.

2. Nédélec Jean-Claude. Acoustic and Electromagnetic Equations. Springer Verlag 2001.

3. Kybic J., Clerc M., Abboud T., Faugeras O., Keriven R., Papadopoulo T.. A Common Formalism for the Integral Formulations of the Forward EEG Problem IEEE Transactions on Medical Imaging. 2005;24:12-28.

4. Geselowitz D. B.. On bioelectric potentials in an homogeneous volume conductor Biophysics Journal. 1967;7:1-11.

5. Hämäläinen M. S., Sarvas J.. Realistic Conductivity Geometry Model of the Human Head for Interpretation of Neuromagnetic Data IEEE Trans. Biomed. Eng.. 1989;36:165-171.

6. Ferguson A. S., Zhang X., Stroink G.. A Complete Linear Discretization for Calculating the Magnetic Field Using the Boundary Element Method IEEE Trans. Biomed. Eng.. 1994;41:455-459.

7. Kybic Jan, Clerc Maureen, Faugeras Olivier, Keriven Renaud, Papadopoulo Théo. Fast Multipole Acceleration of the MEG/EEG Boundary Element Method Physics in Medicine and Biology. 2005;50:46954710.

8. Kybic J., Clerc M., Faugeras O., Keriven R., Papadopoulo T.. Generalized head models for MEG/EEG: boundary element method beyond nested volumes Phys. Med. Biol.. 2006;51:1333-1346.

9. Zhang Zhi. A fast method to compute surface potentials generated by dipoles within multilayer anisotropic spheres Phys. Med. Biol. 1995;40:335-349.

Author: Maureen Clerc

Institute: INRIA

Street: 2004 route des Lucioles, BP93

City: Sophia Antipolis Cedex

Country: France

Email: Maureen.Clerc@sophia.inria.fr 